\title{
The Electric Vehicle Time-of-Use Price Optimization Model Considering the Demand Response
}

\author{
Zejing Shi ${ }^{1,2}$, Ninghui Zhu ${ }^{1,2}$ and Jinsong $\mathrm{Yu}^{1,2}$ \\ ${ }^{1}$ State Grid Electric Power Research Institute, NARI Group Corporation, 211000,Nanjing, China \\ ${ }^{2}$ China EPRI \& Science Technology Co. Ltd., 102200, Beijing, China
}

\begin{abstract}
A large number of electric vehicles connecting to the distribution grids usually introduce significant fluctuations to the grid and the loads. To solve the problem, guiding the users coordinated charging is proposed. Firstly, the uncontrolled charging power prediction models of electric vehicles are established, and the Monte Carlo method is adopted to simulate the power demands of different electric vehicles, and the influences on the load peakvalley ratios and the voltages and losses of the grid are all analyzed. Then the vehicle responses model considering the time-of-use price is analyzed, and the vehicle response ratios are obtained under different time-of-use prices. Finally the multi-objective optimization model is constructed including the minimum peak-valley ratio, maximum consumption satisfaction index and cost satisfaction index. In the procedure, vehicles and the grid are both taken into account. The results indicate the proposed method could guide the users coordinated charging, and the peak shaving and valley filling is also achieved, and the operation of the distribution grid is improved.
\end{abstract}

\section{Introduction}

As the increasing development of electric vehicles and storage systems, the electric vehicles will develop continuously and steadily under the policy encouragement and support. Experts predict that in China, the total number of electric vehicles is about 5,000,000, and simultaneously, 12,000 new charging stations and $4,800,000$ charging poles will be built in 2020 .

The extensive use of electric vehicles is closely related to the planning, constructions and operations of the distribution grid [1-4]. To prevent the grid fluctuations induced by electric vehicles charging, lots of methods are proposed and categorize into directly control method and indirectly guide method. To achieve minimum loss of the grid, reference [5] constructs an optimal model considering the charging power, and iterated revision andTaylor expansion methods are adopted. The minimum load fluctuations and minimum charging costs are both taken into account in [6], and linear weighting method is utilized to normalize the two objective functions. According to the pricing sensitivity model, reference [7] constructs the minimum mean squared error function, and adopts particle swarm optimization method to optimize the time-of-use price. Three kinds of electric vehicles are taken into account in [8], and the charging models are established, and the optimization peak and valley time price project is derived. Reference [9] builds optimization models to minimize the peak-valley ratio, and also constructs the starting moment model of charging considering time-of-use prices, and finally uses the genetic algorithmfor optimization.

Taking private electric vehicles as examples, this paper simulates the influences to the gird during different power electric vehicles charging. Then the vehicle responses to the time-of-use price are analyzed, and the multi-objective optimization model is established depending on the vehicles and the grid. Finally, NSGA((Non-Dominated Sorting in Genetic Algorithms) II is adopted to solve the model equation.

\section{The fluctuations to the grid induced by electric vehicles uncontrolled charging}

\subsection{The simulation of demanded power during the electric vehiclescharging}

The electric vehicle E150 of Baic Motor is considered here, and the parameters are shown in Table 1.

Table 1. The simulation parameters of electric vehicle

\begin{tabular}{|c|c|c|}
\hline $\begin{array}{c}\text { Battery } \\
\text { Capacity/Kwh }\end{array}$ & $\begin{array}{c}\text { Charging } \\
\text { Power /Kw }\end{array}$ & $\begin{array}{c}\text { Maximum daily } \\
\text { mileage/Km }\end{array}$ \\
\hline 25.6 & 3.5 & 130 \\
\hline
\end{tabular}

(1) The charging time and daily mileage 
According to the statistical data of domestic vehicles by US Department of Transportationin 2001, reference [10]derives the daily mileage and returning time of electric vehiclesaccord withthe lognormal distribution and normal distribution respectively through data fitting.

The electric vehicledaily mileaged $(\mathrm{Km})$ comply with lognormal distribution $\mu_{\mathrm{d}}$ of $\sigma_{\mathrm{d}}$ and, the probability density functionis:

$$
f_{d}=\frac{1}{d \sigma_{d} \sqrt{2 \pi}} \exp \left[-\frac{\left(\ln d-\mu_{d}\right)^{2}}{2 \sigma_{d}^{2}}\right]
$$

where $\mu_{t_{s}}=19.5 ; \sigma_{t_{s}}=0.5$

The charging time $t_{s}$ (Assuming returning time of electric vehicles is the charging home) satisfies the normal distribution, and the function is:

$$
f_{t_{s}}=\frac{1}{\sigma_{t_{s}} \sqrt{2 \pi}} \exp \left[-\frac{\left(t_{s}-\mu_{t_{s}}\right)^{2}}{2 \sigma_{t_{s}}^{2}}\right]
$$

where $\mu_{t_{s}}=19.5$;

(2)The charging power of electric vehicles

Monte Carlo method is utilized to derive daily mileagedepending on (1), and consequently, the initial SOC (state of charge) of electric vehicle before charging is also deduced according to (3)

$$
S O C=1-\frac{d}{d_{m}}
$$

According to the initial SOC of electric vehicle,the required time for the electric vehicle fully charged is calculated as:

$$
t_{c}=\frac{(1-S O C) \times C_{n}}{P_{n}}
$$

Then the charging power of a single electric vehicle is shown in (5)

$$
P_{c}=\left\{\begin{array}{lc}
P_{n} & t_{s} \leq t \leq t_{s}+t_{c} \\
0 & \text { others }
\end{array}\right.
$$

(3)The charging power simulation of multiple electricvehicles.

Penetration ratiois defined asthe ratio ofthe charging loads and in distribution grid. The total power of active loads in 33-node distribution network is $3715 \mathrm{~kW}$, if the penetration coefficientis $20 \%$, and consequently, the number of electric vehicles is 212 . The simulation flow chart according to Monte Carlo method is shown in Figure1.

\subsection{The influence analysis on the distribution grid during electric vehicle charging}

By simulations, the loads curves of distribution grid when different power electric vehicles are connected are shown in Figure2.

Figure2 shows as the adding of the electric vehiclepentration ratios, the maximum load increases simultaneously. At the same time, thepeak-valley ratios also increase, resulting inlow efficiency of grid equipment and large grid peaking pressure.
IEEE33-nodesdistribution network is taken as an example here, and the influences on the node voltages and grid losses are both analyzed when large power electric vehicles are connected. The voltage base value is $10 \mathrm{kV}$, and thepower base value is 10MVA.Assuming the charging loads is evenly distributed.

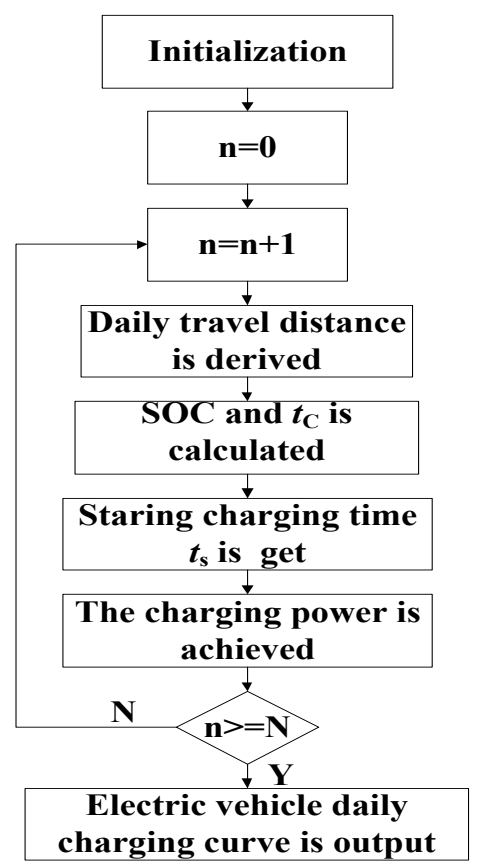

Figure 1. The simulation flowchart by Monte Carlo method.

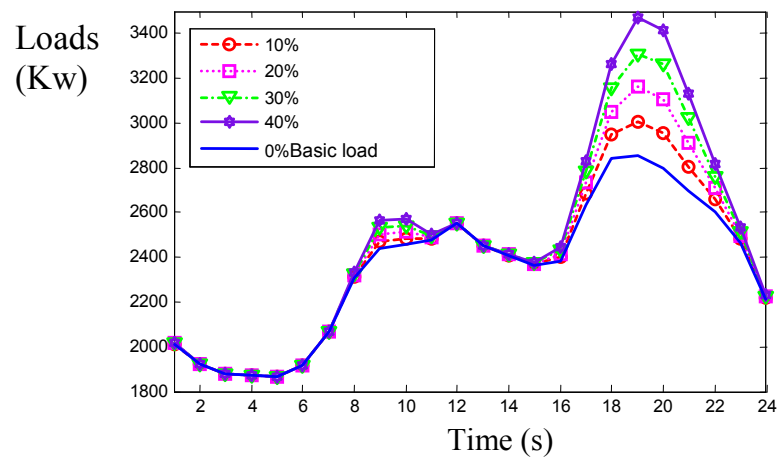

Figure 2. The loads curves of distribution grid with different penetration ratios.

Network loss rate is defined as the ratio of daily network loss and the total load power, and is shown as follows.

$$
R_{p l}=\frac{\sum_{T=1}^{24} P_{\text {loss }}^{T}}{\sum_{T=1}^{24}\left(P_{l+e v}^{T}+P_{\text {loss }}^{T}\right)} \times 100 \%
$$

where $R_{p l}$ is the network loss rate, and $P_{\text {loss }}^{T}$ is the power losses of all networksat the moment $T$, and $P_{l+e v}^{T}+P_{\text {loss }}^{T}$ is the active powerreceived from the source node at the moment $T$.

Figure 3 indicates curves of the voltage per unit value of distribution nodes at 19:00. When the penetration ratios are $0 \%$ and $10 \%$,the voltage amplitudes 
of all nodes are between 0.93 and 1.05 , and consequently, the voltage deviation is allowable. When the penetration ratio is $40 \%$, the voltage amplitudes of nodes $12-18$ and nodes 30-33are all lower than0.93, transcending the limit.

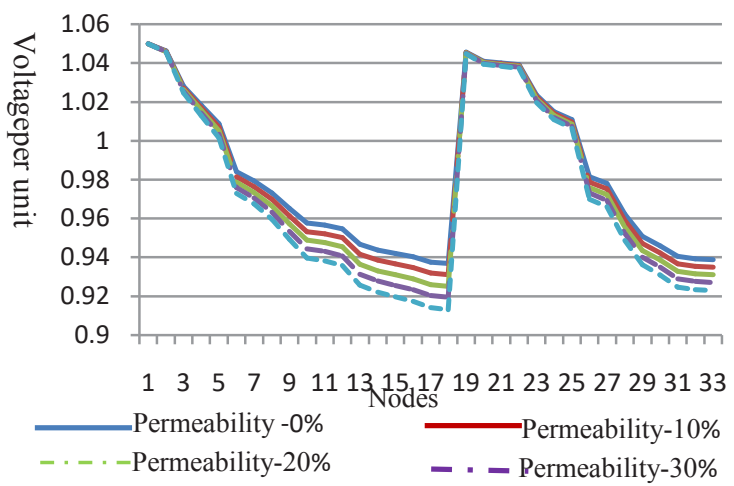

Figure 3. The node voltage curves with different penetration ratios.

Figure 4 shows the grid losses underdifferent penetration ratios. As the increasing of the electric vehicle penetration ratios, the grid losses also increase. The added peak-valley ratios essentially induce large grid losses, which could be derived by Cauchy-Schwarz inequality [11].

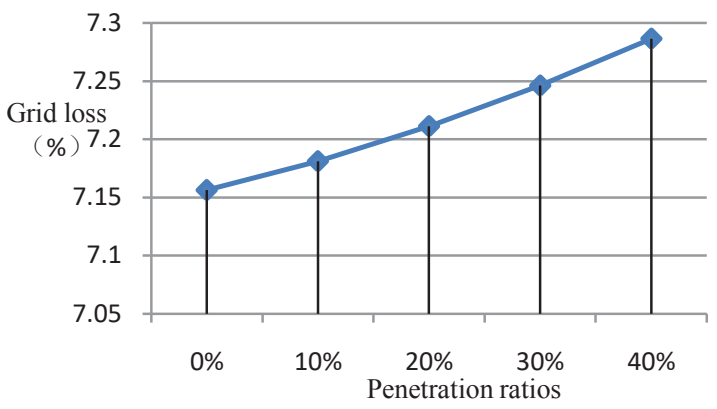

Figure 4. Grid losses with different penetration ratios.

\section{The vehicle responses analysis considering the time-of-use price}

\subsection{The response characteristic curve}

According to consumer psychology, there is a lower threshold in the users consumer stimulus induced by prices, and in this dead zone, the user responses are zero. There is also a saturation value in the userconsumer stimulus, named as saturation zone. Between the dead zone and the saturation zone, there is a linear response zone, and in this area, the relationship between user responses and stimulus is linear. The peak-valley price ratio is defined as stimulus of the response curves, shown in (7), and the response curve is shown in Figure 5.

$$
\beta=\frac{P_{p}-P_{v}}{P_{0}}(7)
$$

where $p_{\mathrm{v}}$ is the valley electricity price, and $P_{\mathrm{p}}$ is the peak electricity price, and $P_{0}$ is the initial electricity price.

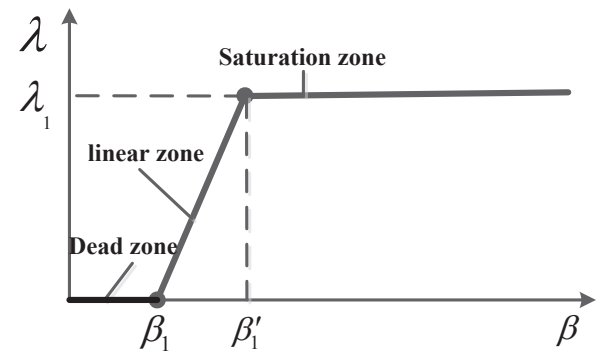

Figure 5. The response curve.

\subsection{The charging time selection of electric vehicles under time-of-use price}

Under time-of-use price, the charging behavior of no response users is not affected, while the response users would make changes to lower the costs. Assuming all the response users would choose a rational charging method, the start time of charging is shown as [10]

$$
t_{s}=\left\{\begin{array}{l}
t_{1}+\operatorname{rand}\left(t_{1}+\Delta t-t_{c}\right) \quad 0 \leq t_{c}<\Delta t \\
t_{1}
\end{array}\right.
$$

where $t_{\mathrm{s}}$ is the start time of charging, and $t_{\mathrm{c}}$ is the charging duration time, and $t_{1}$ is the start time of valley electricity price, while $\Delta t$ is the duration time of valley electricity price.

\section{The optimal charging model of electric vehicles based on vehicles satisfaction index}

The user satisfaction should be fully considered during setting time-of-use electricity prices, and it directly determines the success of the time-of-use prices. If the peak and valley price difference is too large, the charging method of users would change greatly, resulting in hypercorrection. And the great changes may lead to user resistances. On the other side, if the price difference is too little, fewer users would respond and change charging method. Consequently, when setting time-of-use electricity prices, both of users and the grid are supposed to be considered and the equilibrium point is achieved. Depending on the users and the grid, this paper constructs a multi-objective optimization model including the minimum peak-valley ratio, maximum consumption satisfaction index.

\subsection{The objective function}

(1) The equation to minimize the load peak-valley ratio is shown as follows: 


$$
\min L P V\left(t_{s}\right)=1-\frac{\min \left[P_{l+e v}\left(t_{s}, t\right)\right]}{\max \left[P_{l+e v}\left(t_{s}, t\right)\right]}(9)
$$

where LPV is the load peak-valley ratio, and $P_{1+\mathrm{er}}$ represents the power of all loads containing electric vehicles.

(2) Maximize consumption satisfaction index of electric vehicles

Consumption satisfaction indexmentioned here is related to the load curves coefficients before and after the implementation of time-of-use electricity price, and is shown as:

$$
\max \varepsilon=\frac{1}{2} \frac{\sum\left|\left(P_{e v i}^{\prime}-\bar{P}_{e v i}^{\prime}\right)\left(P_{e v i}-\bar{P}_{e v i}\right)\right|}{\sqrt{\left(P_{e v i}^{\prime}-\bar{P}_{e v i}^{\prime}\right)^{2}} \sqrt{\left(P_{e v i}-\bar{P}_{e v i}\right)^{2}}}
$$

where $P_{e v i}, \bar{P}_{e v}$ are the powers of charging loads and average loads at the moment $i$ before time-of-use electricity prices, and $P_{e v i}^{\prime} 、 \bar{P}_{e v}^{\prime}$ are the powers of charging loads and average loads at the moment $i$ after time-of-use electricity prices.

(3) Maximize charging costsatisfaction index

To achieve maximal charging costsatisfaction index, the following is satisfied:

$$
\theta= \begin{cases}0 & C>C_{p} \\ \frac{C_{p}-C}{C_{p}-C_{v}} & C_{v}<C<C_{p} \\ 1 & C<C_{v}\end{cases}
$$

where $C$ is the total charging cost, and $C_{\mathrm{p}} 、 C_{\mathrm{v}}$ are the charging costs when all users choose the peak-price and when they all choose valley-price, respectively.

\subsection{Constraints}

The constraints include the valley beginning time constraint, the valley time duration constraint, peak electricity price constraint, and valley electricity price constraint. These constraints are satisfied:

$$
s t\left\{\begin{array}{l}
t_{s \min }<t_{s}<t_{s \max } \\
\Delta t_{\min }<\Delta t<\Delta t_{\max }(12) \\
P_{p \min }<P_{p}<P_{p \max } \\
P_{v \min }<P_{v}<P_{v \max }
\end{array}\right.
$$

\section{Solving models}

\subsection{NSGA-II algorithm and the procedure}

The main parts of NSGA-II algorithm are fast nondominated sorting and calculating crowding level value [12].

In the fast non-dominated sorting method, each individual has two main parameters: $n_{\mathrm{i}}$ and $S_{\mathrm{i}} . n_{\mathrm{i}}$ is the number of solutions dominating the individuali. $S_{\mathrm{i}}$ is the solutions set dominated by the individuali. The sorting procedure is as follows:

(a) Find the individuals satisfying $n_{i}=0$, and put them into the first level set $F_{1}$.

(b) Find the individual set $S_{j}$ dominated by each individual $j$ in $F_{1}$, and subtract 1 from the $n_{k}$ of every individual $k$, if satisfy $n_{k}-1=0$, putthe individual $k$ into $F_{2}$.

(c) Set $F_{1}$ asthe first non-dominated individuals set, and all the individuals in $F_{1}$ are the optimal individuals, and these individualsare not dominated. Each individual isgiven to a non-dominated sequence $i_{\text {rank }}$.

(d) Repeatstep (b) and step (c)in $F_{2}$, until the individuals are all graded and are alsogiven to thenondominated sequences $i_{\text {rank }}$.

Crowding levelis used to evaluate the density of other individuals around anindividual, and it could keep the populationdiverse, and prevent partial accumulation problem. The schematic diagram of the crowding level is shown in Figure 6.

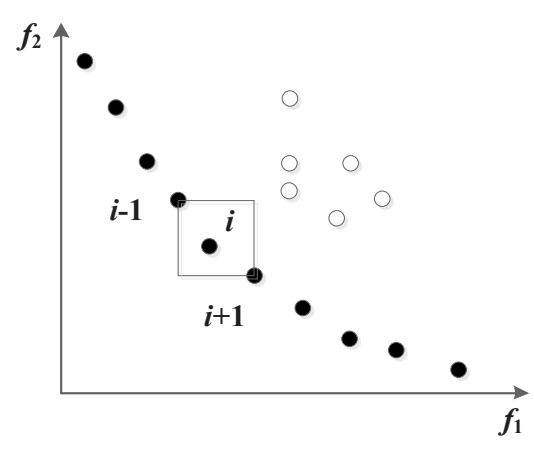

Figure 6. The schematic diagram of the crowding level.

The crowding level value of individual is calculated as

$$
i_{d}=\frac{\sum_{j=1}^{m}\left(\left|f_{j}^{i+1}-f_{j}^{i-1}\right|\right)}{f_{j}^{\max }-f_{j}^{\min }}
$$

where $f_{j}^{i+1} 、 f_{j}^{i-1}$ are the $j$ objective function values of individual $i+1$ and $i-1$, respectively, and $m$ is the number of objective functions. The crowding level values of the boundary individualsare defined as infinity. 
The main flowchart of NSGA- II algorithm is shown in Figure 7.

The initial population $P_{t}$ of size $\mathrm{N}$ is randomly generated, and a new offspring population $Q_{t}$ is produced by genetic manipulation. The initial population and offspring population are combined together and achieve a new population $R_{t}=P_{t} \cup Q_{t}$. Thenon-dominated sorting is performed in $R_{t}$, and the crowding levels of individuals in $F_{i}$ arecalculated. Then the best individual in $R_{t}$ isselected.All individuals in $F_{1}$ are put into a new parent population $P_{t+1}$, if the number of individuals innew population $P_{t+1}$ is less than $\mathrm{N}$, then the individuals of next layer are placed into it. Otherwise, if the number of individuals inpopulation $P_{t+1}$ is greater than $\mathrm{N}$, the individuals choosing is performedaccording to the individual crowing level to ensure the number of individuals is N. Genetic manipulationproduces new offspring population $Q_{t+1}$ from the new parent population $P_{\mathrm{t}+1}$.The procedure is repeated until the algorithmsatisfies certain constraints.

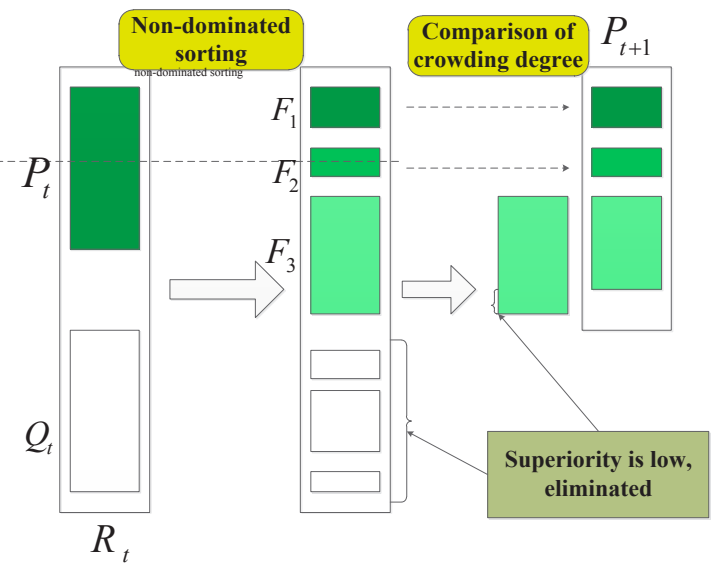

Figure 7. The main flowchart of NSGA- II algorithm.

\subsection{The optimal compromise solution}

The multi-objective solution is not an optimal solution, but a non-dominated solution set. The optimal compromise solution is needed to be chosen from this set. Partial membership function is used to achieve the optimal compromise solution, and shown as [9]

$$
u_{i}= \begin{cases}1 & f_{i}<f_{i}^{\min } \\ \frac{f_{i}^{\max }-f_{i}}{f_{i}^{\max }-f_{i}^{\min }} & f_{i}^{\min }<f_{i}<f_{i}^{\max } \\ 0 & f_{i}>f_{i}^{\max }\end{cases}
$$

where $u_{i}$ is the satisfaction index of the individual objective function $i$, and $f_{i}^{\text {min }} 、 f_{i}^{\text {max }}$ are the minimum value and maximum value of the objective function $i$, respectively.

The individual satisfactionindex in Pareto solution set is calculated as (15). The individual with maximum $u$ is selectedas the optimal compromise solution.

$$
u=\frac{1}{m} \sum_{i=1}^{m} u_{i}
$$

\subsection{Examples}

Derived Pareto optimal solution set is shown in Figure 8.The content of Figure 8 indicates the Pareto optimal solution set has excellent diversity, and is evenly distributed. Consequently, the optimal model proposed by the algorithm has a strong global search capability. The derived optimal compromise solutionsare shown in Table 2 .

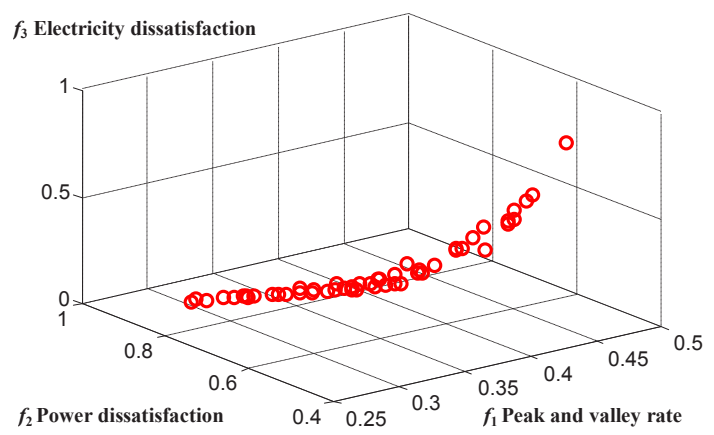

Figure 8. Paretooptimal solutionset.

Table 2. The optimal compromise solutions

\begin{tabular}{|c|c|c|c|}
\hline$t_{s}$ & $\begin{array}{c}\Delta t \\
\text { (hour) }\end{array}$ & $\begin{array}{c}\mathrm{v} \\
\text { (Yuan) }\end{array}$ & $\begin{array}{c}P_{v} \\
\text { (Yuan) }\end{array}$ \\
\hline & 11 & 1.0449 & 0.5056 \\
\hline
\end{tabular}

The derived objective function values of optimal compromise solutions are in Table 3. The optimal compromise solution tends to decreasethe peak-valley ratios of the grid and gets low consumption satisfaction index, but the costsatisfaction index is very high, and consequently, the user responseratiois also high.

Figure 9 shows the load power comparison curves under two charging modes, and it indicates the optimized charging loads play an important role in the peak clipping and valley filling of distribution grid.

Figure 10 gives the node voltage per unit valuesunder two charging modes. After optimized charging, the quality of voltage is improved, and the voltages of nodes 17 and 18 are both higher than 0.93 , which satisfies the voltage requirement of $10 \mathrm{kV}$ grid. 
Table 3. The objective function values of optimal compromise solutions

\begin{tabular}{|c|c|c|c|}
\hline $\begin{array}{c}\text { Response } \\
\text { ratio } \\
(\%)\end{array}$ & $\begin{array}{c}\text { Peak-valley } \\
\text { ratio (\%) }\end{array}$ & $\begin{array}{c}\text { Consumption } \\
\text { satisfaction } \\
\text { index }\end{array}$ & $\begin{array}{c}\text { Cost } \\
\text { satisfaction } \\
\text { index }\end{array}$ \\
\hline 76.88 & 33.76 & 0.6806 & 0.7689 \\
\hline
\end{tabular}

\section{Conclusion}

Firstly, this paper analyzes the fluctuations to the grid during different electric vehicles charging. Then depending on the vehicle responses, the minimum peakvalley ratio and maximum satisfaction index optimization model is constructed considering both of the gird and vehicles. Finally, NSGA- II algorithm is utilized to solve the model equation. The analysis indicates:

1. In the optimized charging mode, the electric vehicles play an important rolein peak clipping and valley filling procedure, and also could improve efficiency of grid equipment.

2. In the optimized charging mode, the quality of the grid voltage is improved significantly, and losses of the grid are decreased.

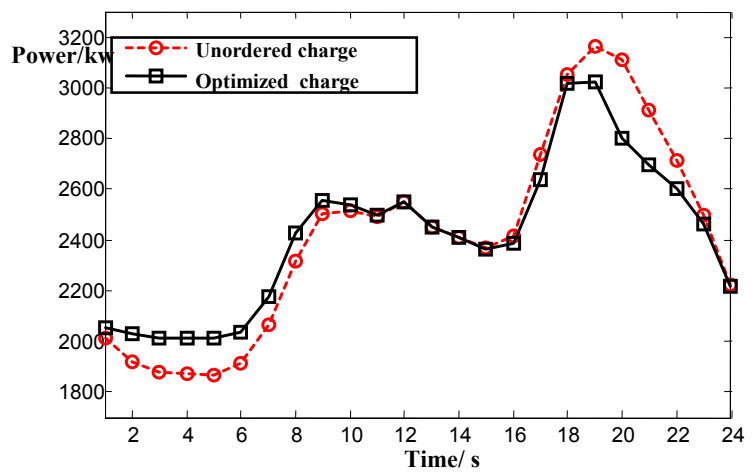

Figure 9. The load power comparison curves under two charging modes.

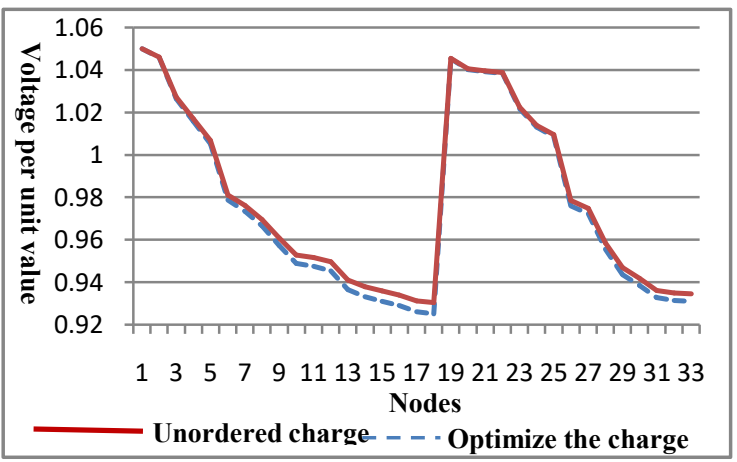

Figure 10. The node voltage per unit valueunder two charging modes.

The vehicle response ratio is only affected by time-of-use price in this paper, but unfortunately, in practical applications, many factors influence the vehicle responses, such as financial conditions and charging implementations. The accuracy of the response curves needs to be improved in the future.

\section{Reference}

1. HU Zechun, SONG Yonghua. Impacts and Utilization of Electric Vehicles Integration Into Power Systems $[\mathrm{J}]$. Proceeding of the CSEE. 2012, 32(4); 1-10

2. Taylor M J, Alexander A. Evaluation of the impact of plug-in electric vehicle loading on distribution system operation [C] //IEEE power \&Energy Society General Meeting, Calgary, Canada, 2009: 1-6.

3. Kejun Q, Chengke Z, Allan M, et al. Modeling of load demand due to EV battery charging in distribution systems[J] . IEEE Transactions on Power Systems, 26(2): 802-810.

4. Kristien $\mathrm{CN}, \mathrm{EH}$, johan D. The Impact of Charging Plug-In Hybrid Electric Vehicles on a Residential Distribution Grid[J]. IEEE Trans on power systems, 2010 (255):371-380.

5. ZHAN Kaiqiao. SONG Yonghua, Coordination of Electric Vehicle Charging to Minimize Active Power Losses[J], Proceedings of the CSEE, 2012, 32(31): $11-18$

6. NI Feng, ZHAO Ming-yu, Research on ordered charging of EV [J], Power Demand Side Management, 2015, 17(5): 16-19

7. LUO Min, ZHAO Wei, Research on Coordinated Charging Price of Electric Vehicle Based on Users' Price Sensitivity [J], Electrical \& Energy Management Technology, 2015: 78-92.

8. GAO Yajing, LYU Mengkuo, Power Demand Price Elasticity Matrix Based on Discrete Attraction Model[J], Automation of Electric Power Systems, 2014, 38(13):103-107.

9. GE Shao-yun, HUANG Liu, Optimization of peakvalley TOU power price time-period in ordered charging mode of electric vehicle $[\mathrm{J}]$. Power System Protection and Control , 2012, 40(10):1-5.

10. GONG Xin, LIN Tao. Impact of Plug-in Hybrid Electric Vehicle Charging on Power Distribution Network [J]. Power System Technology. 2012, 36(11): 30-35

11. CHEN Jia-sheng, ZHANG Jian-hua, Strategies for Electric Vehicle Charging with Aiming at Rwducing Network Losses[J]. Proceedings of the CSU-EPSA, 2012,24 (3): :139-144.

12. GAO Yuan, Non-dominated Sorting Genetic Algorithm and Its Applications [D]. Zhejiang University, 2006 